\title{
Large Format FDM Printing of Recycled Plastic Pellets: Closing Consumer Cycles in the fabrication of meso scale objects
}

\author{
Rodrigo Shiordia López \\ Universidad Anáhuac | México | rodrigo.shiordia@anahuac.mx
}

\begin{abstract}
Fused Material Deposition (FDM) is a widely used technology for desktop 3d printing which has the potential to close consumer cycles by printing recycled materials. However, its use in architectural scale applications has still to be further explored. This paper presents the design, construction and implementation of a $3 d$ printing system of recycled plastic pellets. We describe the device, its systems and parameters, as well as the custom tool path calculations and material experiments. We argue for this technology as a viable way of implementing FDM on larger scales and identify key challenges that present themselves as we explore the fabrication of meso scale formal artifacts.
\end{abstract}

Keywords: 3D printing; FDM Printing; Recycled materials; digital and robotic fabrication; additive manufacturing.

\section{INTRODUCCIÓN}

La impresión 3d representa una de las tecnologías para la producción de objetos y componentes arquitectónicos con el mayor potencial de impactar positivamente en la industria de la construcción. En la última década se han visto avances sustantivos en esta tecnología, desde su adopción como una de las tecnologías más usadas en Fablabs y talleres de fabricación digital académicos y comerciales, hasta su desarrollo como una tecnología que permita la creación de espacios arquitectónicos.

Los plásticos en arquitectura se han estudiado desde la década de los '60s (Borden \& Meredith, 2012), sin embargo, es en recientes años que la impresión 3d presenta oportunidades para su aplicación en contextos arquitectónicos.

Un subgrupo de tecnologías de impresión 3d ampliamente disponible es el que implementa el principio de deposición de material fundido (FDM por sus siglas en inglés). Esta tecnología utiliza energía térmica proporcionada por resistencias eléctricas para fundir materiales. normalmente termoplásticos - a través de un extrusor. Esta tecnología es la más utilizada en equipos de bajo costo y comerciales por su practicidad y facilidad de uso.

Mientras que ya existen equipos con alto refinamiento en el control y calidad de las impresiones, el reto persiste en términos de escala. La tecnología FDM sigue siendo una tecnología cuyas capacidades se encuentran limitadas por la dificultad que representa la impresión $3 \mathrm{~d}$ de gran formato. Las impresoras $3 \mathrm{~d}$ comerciales están diseñadas con volúmenes de impresión menores a los 30×30 o 40x40 centímetros como máximo. Esto supone una gran dificultad para usarlas en aplicaciones arquitectónicas y componentes de mayor escala.
Adicionalmente, la tecnología FDM mantiene una dependencia en la implementación de filamento de plástico, que es el insumo principal del sistema. El filamento de plástico supone una fácil implementación de la tecnología, pero es un insumo que limita el tamaño de impresión, debido a los diámetros utilizables.

El plástico sigue siendo uno de los materiales más utilizados en la industria de consumo y existen grandes cantidades de desperdicio de este material. Las universidades, laboratorios, y espacios de fabricación digital no son la excepción en términos de producción de desperdicios plásticos. Debido a las particularidades de la tecnología FDM, normalmente se utiliza material de soporte para el apuntalamiento de objetos a la hora de imprimirlos. Este material normalmente es desechado. Mientras que cada impresión utiliza pocas cantidades de material de soporte, este material es una fuente de bajo costo para reciclaje de termoplásticos. Los objetos impresos por impresoras $3 \mathrm{~d}$, así como los materiales de soporte usados para apuntalar las impresiones y objetos de consumo de corta vida, siguen siendo fuentes de desperdicio plástico que deben de ser reciclados. Para mitigar el impacto ambiental de los plásticos en nuestro planeta, es necesario cerrar ciclos de consumo localmente, reciclando cualquier fuente de desperdicios plásticos ahí donde se utilizan.

Para poder extender las aplicaciones de la tecnología FDM hacia el ámbito arquitectónico, es necesario que esta tecnología dé un brinco en términos de escala. Aunque existen interesantes implementaciones de tecnología FDM a la escala del espacio construido, (Rael \& San Fratello, 2018) las limitaciones, principalmente en términos de escala de estos sistemas, constituyen uno de los mayores retos para su adopción en la construcción. 


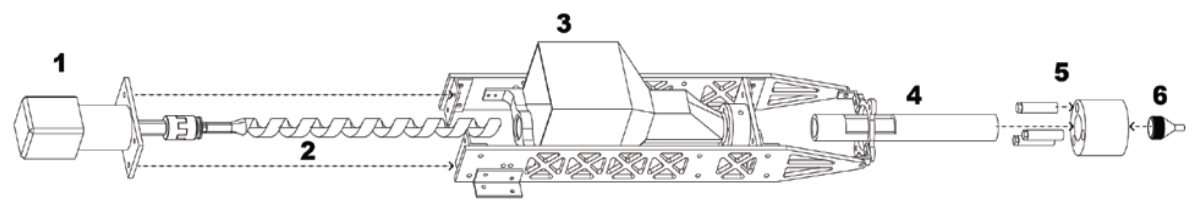

Figura 1: El diseño del dispositivo muestra el motor con el husillo (1 y 2), el depósito de pellets (3), el tubo de alimentación con su abertura lateral (4), el bloque de calor con los cartuchos de resistencia (5), y la boquilla de salida (6).

Este trabajo presenta el diseño, fabricación, operación e implementación de un dispositivo de bajo costo para la impresión $3 \mathrm{~d}$ de gran formato de objetos con potenciales usos arquitectónicos a partir del reciclaje de desechos termoplásticos comunes en laboratorios de fabricación digital. Se introduce el dispositivo diseñado y producido por el autor y un equipo de investigadores independientes para la impresión $3 \mathrm{~d}$ a gran escala de material plástico reciclado directamente de objetos post-consumo y de material de soporte de impresiones $3 \mathrm{~d}$, acumulado durante años de impresiones en diversos laboratorios. El sistema presentado es capaz de producir objetos de mayor escala que impresoras $3 \mathrm{~d}$ comunes de manera confiable y rápida, gracias a decisiones clave en el diseño y operación del dispositivo. Este trabajo se concentra en el sistema de extrusión, la última etapa en un sistema de impresión 3d. El sistema de movimiento y control fue proporcionado por un brazo robótico Kuka KR30, pero la trayectoria del extrusor puede ser proporcionada por otro mecanismo del tipo gantry más común y con un mucho menor costo. El enfoque de este trabajo es la discusión de la etapa de extrusión. Se discutirán importantes decisiones tomadas en el diseño de las trayectorias de impresión, y algunos de estos aspectos tendrán relación con las capacidades y limitantes del sistema de movilidad por medio de brazo robótico, sin embargo, se tratará de generalizar estos aspectos como parte de un sistema de movilidad genérico de los cuales existen sistemas factibles (Ingrassia, 2020).

Este trabajo también propone el ámbito de la escala meso como la opción más viable para la tecnología presentada. La escala meso es la escala de objetos entre el objeto de consumo, y el objeto arquitectónico. El objeto meso opera en la escala mayor que la de la mano o el brazo humanos, en la cual operan la mayoría de las impresoras 3d comerciales o "desktop". Esta escala es menor que el espacio arquitectónico, en donde operan sistemas de impresión $3 \mathrm{~d}$ de barros, adobes y concretos. La escala meso, es la escala del mueble, la cual se discute a través de ejemplos y resultados obtenidos, como el punto idóneo para el uso de impresión 3d utilizando termoplásticos.

\section{DISEÑO DEL DISPOSITIVO}

\section{SISTEMAS DEL DISPOSITIVO}

El dispositivo tiene tres partes importantes, lo que llamamos el sistema termoeléctrico, el sistema electromecánico y el chasis de montaje.

El sistema electromecánico comienza en un motor NEMA23 con una transmisión planetaria de 47:1 revoluciones que opera a un voltaje de 50 Volts y una corriente de 2.7 Amperes. Este motor hace girar un husillo de $23 \mathrm{~mm}$ de diámetro dentro de un tubo metálico con apertura lateral para la alimentación de pellets de plástico. El motor modula su velocidad gracias a un driver y un micro controlador, que son programados para dar velocidades desde las 11rpm hasta las 30rpm. Es importante mencionar que el motor tiene una transmisión planetaria de 47:1 revoluciones, lo que aumenta considerablemente el torque, y disminuye su velocidad. La apertura lateral del tubo se encuentra fijada a un depósito impreso en $3 \mathrm{~d}$ que concentra y dirige los pellets al husillo para ser transportados y comprimidos al entrar en el sistema termoeléctrico.

El sistema termoeléctrico se compone de un bloque de calor a de aluminio con tres resistencias de 75 watts que calientan el material hasta temperaturas cerca de los $300^{\circ} \mathrm{C}$ y que dirige el material a una boquilla de bronce que reduce el diámetro de la cavidad de $23 \mathrm{~mm}$ a $3 \mathrm{~mm}$. Las resistencias eléctricas se encuentran controladas por un pirómetro de control Proporcional, Integral y Derivativo (PID) que mantiene la temperatura constante y permite aumentar o reducir la temperatura del bloque de calor.

Ambos sistemas se encuentran fijados a un chasis de aluminio que mantiene los componentes en su sitio y transmite las fuerzas del motor al husillo, constriñendo los grados de libertad del motor para una operación segura y eficiente. El chasis permite que el dispositivo sea fijado al flange del brazo robótico o en su caso al carro de una impresora con gantry.

\section{DIÁMETRO DE LA BOQUIILLA DE EXTRUSIÓN}

Desde un principio se tenía el objetivo de buscar la impresión 3d de objetos de gran escala. Para poder hacer la impresión $3 \mathrm{~d}$ a gran escala posible, la primera decisión que se tomó fue la del diámetro de la boquilla de extrusión. Por lo general, los diámetros de las boquillas de extrusión de sistemas de impresión $3 \mathrm{~d}$ rondan el orden de los 0.4 a los 0.7 milímetros. El diámetro de la boquilla va relacionado al nivel de detalle y las características más finas que se pueden imprimir. En el caso de la impresión a gran escala, es necesario aumentar el diámetro de la boquilla para lograr velocidades de impresión viables. De lo contrario, el tiempo de impresión podría ser prohibitivo.

Debido al aumento en el diámetro de la boquilla, los filamentos comunes no pueden usarse directamente en la impresión $3 d$ a gran escala con velocidades viables. Los filamentos comunes en impresoras $3 \mathrm{~d}$ son del orden de los 1.75 a 2.85 milímetros de diámetro. Una de las necesidades de la impresión $3 \mathrm{~d}$, es que al ser extruido, el plástico debe de pasar por una cavidad cónica a grandes temperaturas, lo que permite su compresión para poder 
mantener una impresión uniforme. Debido a esto, la boquilla de impresión siempre debe de ser menor al diámetro del filamento de impresión. Cuando se toma la decisión de utilizar diámetros mayores a los 3 milímetros en nuestras boquillas, se debe de buscar una alternativa al filamento. Nuestra propuesta desde un principio tenía que utilizar ya sea un filamento con un diámetro mayor, o utilizar otro tipo de insumo para la extrusión. En el dispositivo se probaron diámetros de 6, 5, 4 y 3 milímetros de extrusión, siendo el de $3 \mathrm{~mm}$ el que mostró más consistencia en la impresión.

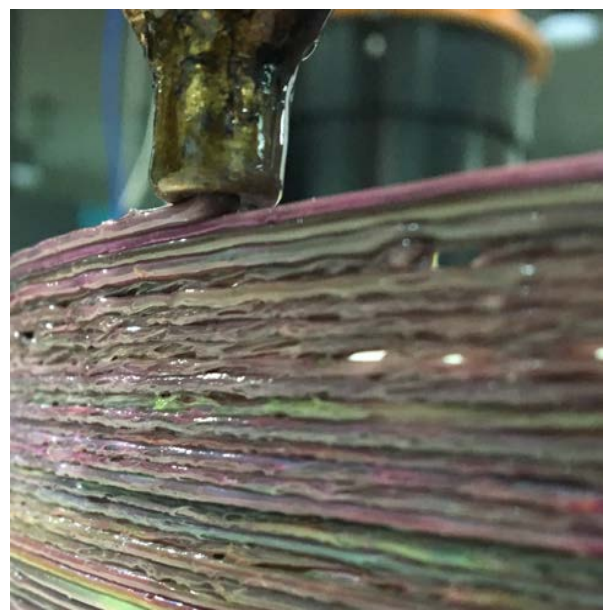

Figura 2: La boquilla de extrusión es fundamental para la impresión. Aparte del diámetro, la longitud de la cavidad interior contribuye a la consistencia de extrusión.

\section{IMPRESIÓN DE PELLETS DE PLÁSTICO: CERRANDO CICLOS DE CONSUMO}

Debido al diámetro objetivo de extrusión, se optó por utilizar pellets o micro esferas de plástico como insumo para el dispositivo de extrusión. Debido a la gran cantidad de desperdicio de impresiones que se ha acumulado en el laboratorio de fabricación digital de nuestro laboratorio, se optó por usar este mismo material como el insumo para el dispositivo. Se contaba con cerca de 50 kilogramos de impresiones fallidas, material de soporte, y pruebas descartadas de plástico PLA (ácido poli-láctico por sus siglas en inglés, un material muy común en los filamentos de impresión comerciales). Este material fue molido en una maquina astilladora de madera, cernido en diferentes tamaños de mallas metálicas, para lograr partículas de hasta $2 \mathrm{~mm}$ de tamaño. Este material era multicolor, por lo que no se separaron los colores, y la mezcla de ellos mostraba un color morado que se decidió era adecuado para nuestros objetivos.

El material molido con este método es una gran oportunidad para cerrar ciclos de consumo en Fablabs, makerspaces y laboratorios de fabricación digital. Quien opere uno de estos espacios podrá ver que siempre hay impresiones descartadas, material de soporte que es desechado después de ser removido de las partes impresas, impresiones fallidas que no terminan de imprimirse, etc. Una estrategia de recuperación para reciclaje de este material permite que este plástico no llegue a nuestros océanos y se utilice para generar más objetos de consumo para mercados pequeños y especializados, y en un punto ideal, para componentes arquitectónicos de escala real y de uso final.

Adicionalmente, los pellets de plástico son una forma común de comercialización de termoplásticos, ya que son el insumo principal para hacer moldeo por inyección, y la industria tiene disponibles estos materiales para su consumo. Se probaron también mezclas de material reciclado con resinas plásticas vírgenes o de primer uso.

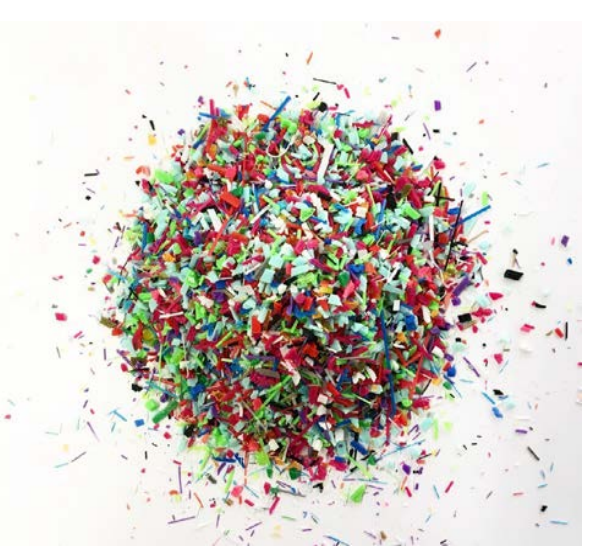

Figura 3: El material fue molido y cernido hasta lograr un tamaño de partícula menor a los $2 \mathrm{~mm}$.

\section{DISEÑO DE TRAYECTORIAS PARA EL EFECTOR}

El movimiento del dispositivo lo proporciona un brazo robótico Kuka. El dispositivo ha sido utilizado en un modelo KR30 y un modelo KR10. Los sistemas del dispositivo que van fijados al sistema de trayectorias tan sólo pesan 7800 gramos por lo que su uso en diversos sistemas que proporcionen movimiento es viable.

Para el cálculo de las trayectorias de impresión se utilizó Grasshopper para la obtención de los contornos de impresión con la opción de controlar la altura entre capas. Se utilizaron capas de 1, 1.5 y 2 milímetros, lo cual permite imprimir a velocidades mayores. En impresoras 3d comerciales de filamento, la altura de la capa oscila entre los $0.1 \mathrm{~mm}$ y los $0.5 \mathrm{~mm}$. La diferencia en alturas de capa es uno de los parámetros clave que hacen de la impresión de material fundido una opción viable para objetos a escalas mayores. A partir de un cálculo de contornos a la altura deseada, se debe de producir un solo objeto lineal de impresión dado que el control de encendido y apagado de la extrusión aún no ha sido desarrollado. Este objeto lineal debe de contener la geometría del objeto así como los saltos entre capa y capa.

En una primera versión, se utilizó el sistema de escalón, en donde la diferencia de altura entre cada capa es absorbida por un "escalón" en el último segmento de la trayectoria de la capa. Este sistema es eficiente y presenta 
buena adhesión entre las capas, pero presenta secciones de la superficie del objeto impreso que pueden no ser estéticas. Adicionalmente, el escalón debe de ser calculado de tal manera que pueda estar exactamente por encima del mismo punto de la capa inferior, lo cual se optimizó con la intersección entre las curvas de las capas, y un plano vertical que proporcionó los puntos finales de las trayectorias de cada capa.

Para resolver el problema del "escalón" entre capa y capa se optó por el desarrollo de una definición de Grasshopper que simula el "vase mode" o modo "florero" en el que la altura entre cada capa está dividida entre cada punto de la trayectoria, mostrando así una línea de impresión óptima sin detalles indeseables en la superficie del objeto.

Los dos métodos son ideales para controlar los parámetros de impresión de una manera sencilla, pero a la vez manteniendo el control que un programa estándar de rebanado o slicing simplemente no brinda pues son algoritmos que normalmente no ofrecen buenos parámetros de control al usuario. El ambiente Grasshopper es ideal para controlar la geometría de las trayectorias a un nivel más avanzado.

Una vez que se cuenta con la geometría de la trayectoria, se debe de enviar al robot por medio de un archivo *.src que es producido por un plugin especial. Se utilizó el plugin Kuka PRC Parametric Robot Control (Asoc. For Robots in Architecture, 2020) para los cálculos cinemáticos del robot. Este toma como entrada una serie de planos que representan puntos sobre la trayectoria deseada. Los puntos, al ser planos incluyen la información de posición y orientación del robot al mismo tiempo.

\section{TEXTURIZADO}

En el diseño de las trayectorias de impresión, se aprovechó el control que se tiene para generar geometrías que pueden llegar a ser difíciles de lograr en un flujo de trabajo convencional. En el flujo de trabajo convencional, el usuario genera un modelo $3 d$ que es exportado en un formato de mala hermética, que después es rebanado y contorneado por un software que se encarga de calcular las trayectorias. Nuestro flujo de trabajo permite al usuario con un nivel intermedio o avanzado de Grasshopper un control más específico de las trayectorias. Una vez calculadas las trayectorias, se experimentó con trayectorias que presentan desfases en cada segunda 0 tercera capa.

Estos desfases permiten un diseño gráfico de texturas que aumenten el espesor de la pared sin modelar explícitamente estos detalles. Nuestro sistema de texturizado opera directamente en la trayectoria de impresión, dividiendo cada capa en un número dado de puntos, se puede tener un espacio de dos dimensiones, una dimensión la posición interpolada del punto sobre la curva del contorno de la capa, y la segunda dimensión la altura o el número de cada contada desde el origen. De esta manera se pueden implementar cualquier tipo de algoritmos de texturizado, desde muestreo de imágenes hasta difusiones reactivas que permitan imprimir y codificar espesores y texturas específicos y complejos en la impresión sin ser modelados explícitamente, esta operación puede tomar como base cualquier geometría y efectuarse de manera rápida pues opera en un nivel de abstracción alto codificando el espacio paramétrico de la superficie a la hora de fabricación.

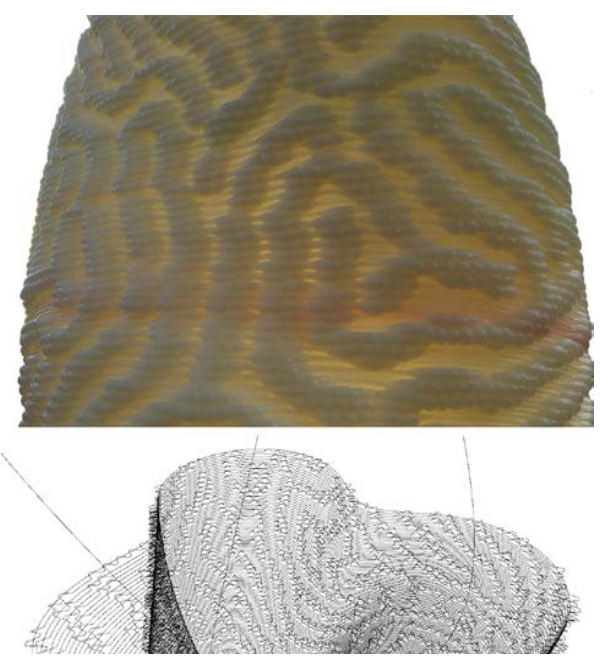

Figura 4: El texturizado se logra interviniendo directamente las trayectorias de la capas, evitando el modelado explícito de estos detalles.

\section{TRAYECTORIAS TRIDIMENSIONALES}

El alto control que tenemos sobre las trayectorias, permite fácilmente generar capas que son completamente planas. Se diseñaron algunas trayectorias con un control también sobre el eje $Z$ del contorno de la capa, permitiendo así el diseño de texturas y diferenciales en alturas que no sería posible con sistemas comerciales. Estas trayectorias pueden ser optimizadas para lograr concentraciones de material en donde sea necesario para coadyuvar en el desempeño estructural, concentrando material donde se concentren estreses térmicos o estructurales.

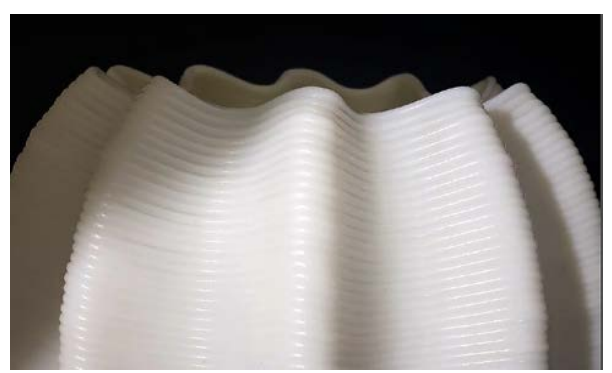

Figura 5: Gracias al control de la geometría de la trayectoria se generaron capas que transitan de totalmente planas a trayectorias tridimensionales.

\section{DISEÑO DE ARTEFACTOS DE ESCALA MESO}

En su forma actual, el sistema no incluye el control de inicio y final de extrusión, por lo que los objetos impresos con nuestro sistema deben de ser topológicamente cilíndricos, permitiendo que se impriman en una sola trayectoria, ya sea en espiral o escalonada. Más que ver esta condicionante como una limitación, se toma como un inicio para el diseño de este tipo de objetos. 
Como se ha mencionado, la impresión FDM presenta importantes retos para su viabilidad en la impresión de gran formato. Nuestro sistema opera óptimamente con capas de altura de $1.5 \mathrm{~mm}$ y boquilla de extrusión de $3 \mathrm{~mm}$. Estas dimensiones son prometedoras para extrusiones mayores a las que son posibles con dispositivos comerciales, sin embargo no son viables aún para la impresión completa de espacios arquitectónicos. Esta condición representa el punto de partida para nuestra propuesta de conceptualizar la escala meso como la opción más viable para esta tecnología. Nuestros objetos pueden imprimirse con buenas velocidades y con consistencia en la escala entre los objetos que pueden caber en la mano humana (la escala micro), y los objetos en los que cabe el cuerpo humano (la escala macro).

La escala micro es la escala en donde operan los sistemas de impresión $3 \mathrm{~d}$ comerciales, con volúmenes de hasta $300 \times 300 \times 300 \mathrm{~mm}$. La escala macro es la escala en la que operan los sistemas de impresión 3d de concreto y otros materiales, que son capaces de imprimir en $3 d$ espacios arquitectónicos donde el cuerpo cabe completamente, el volumen de esta escala ronda los $3000 \times 3000 \times 3000 \mathrm{~mm}$. La escala meso, opera entre estas dos escalas, y debido a las limitantes del sistema de impresión FDM, es importante que los objetos se diseñen para esta escala. Nuestros volúmenes de impresión, son viables alrededor de los $1000 \times 1000 \times 1000 \mathrm{~mm}$. Esta escala, mientras que no es suficiente para la impresión de arquitecturas, es altamente recomendable para muebles y componentes arquitectónicos.

\section{RESULTADOS}

\section{USUARIOS}

Nuestro sistema fue implementado con diseños propios así como con diseños de alumnos de un programa de posgrado en arquitectura, con los cuales se compartió el flujo de trabajo, así como la operación del dispositivo. Se experimentaron diferentes estrategias para el diseño de las trayectorias de impresión, mismas que hemos discutido con anterioridad.

\section{GEOMETRÍA}

La mayoría de los objetos impresos topológicamente son cilíndricos con un alto grado de variabilidad en términos de geometría. El objeto más grande aún impreso tiene una altura de $600 \mathrm{~mm}$. Los diámetros de impresión más manejables se encuentran alrededor de los $600 \mathrm{~mm}$. Se produjeron boquillas de extrusión de 6,54 y $3 \mathrm{~mm}$ de diámetro. Las boquillas con diámetros mayores tendieron a presentar mayores imperfecciones en la extrusión, pero permiten alturas de capa mayores. Las boquillas con menores diámetros presentan una extrusión constante y con menos imperfecciones, sin embargo, las alturas de capa son menores, lo que supone mayores tiempos de impresión. Nuestro objetivo siempre fue minimizar los tiempos de impresión, para poder maximizar los tamaños de los objetos impresos, por lo que la altura de capa siempre fue un parámetro clave. Comparativamente, la boquilla de $3 \mathrm{~mm}$ con alturas de capa de $1.5 \mathrm{~mm}$ fue la de más alto desempeño en términos de la calidad y velocidad de impresión.

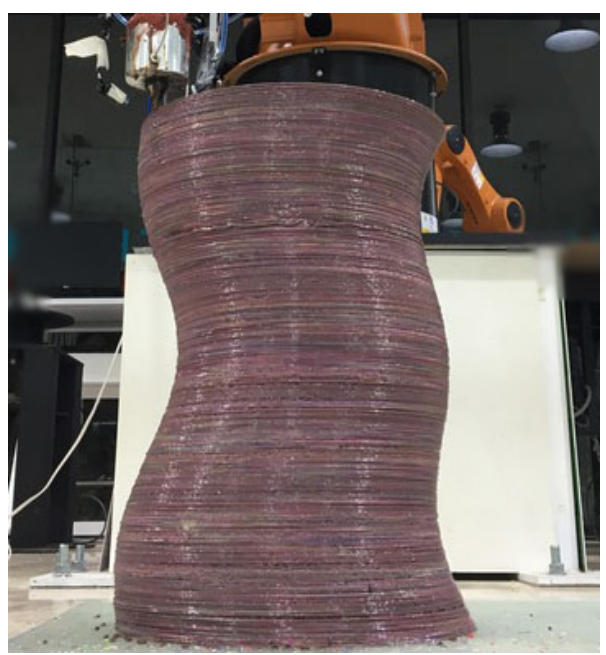

Figura 6: El diseño e impresión de objetos de escala meso es la implementación más viable de nuestro sistema, el artefacto mide $40 \mathrm{~cm}$ de diámetro, por $60 \mathrm{~cm}$ de altura.

\section{MATERIALES}

Se utilizaron los plásticos ABS, PLA, PET y HDPE, de los cuales, a continuación se discute cada uno y sus ventajas y desventajas para su uso en impresión directa desde pellet de plástico. Sin duda, el plástico PLA es el más sencillo de usar por su bajo coeficiente de encogimiento térmico, su fácil adherencia entre capas y su baja temperatura de extrusión $\left(195^{\circ} \mathrm{C}\right)$. Sin embargo, el PLA es de los plásticos menos dúctiles, por lo que las partículas deben de ser molidas hasta tamaños menores para evitar atascos en el sistema. El ABS, otro material común en filamentos de impresión $3 d$, presentó fuertes problemas de encogimiento térmico y adherencia entre capas. Para mejorar la adherencia de capas, se aumentó la temperatura de extrusión hasta $230^{\circ} \mathrm{C}$. El uso de un ambiente con temperatura controlada por medio de una cama caliente y una pistola de aire caliente fueron dos factores que minimizaron el encogimiento térmico. El polietileno de alta densidad (HDPE) es un plástico muy común en envases y bolsas desechables. Este material presenta encogimientos térmicos dramáticos que hacen su uso prohibitivo para impresión $3 \mathrm{~d}$ tradicional. Sin embargo se experimentó con trayectorias tridimensionales que mientras que minimizan los puntos de contacto entre capa y capa. También se mezclaron diferentes proporciones de estos materiales, pero esto no es recomendable ya que la adherencia entre capas disminuye. 


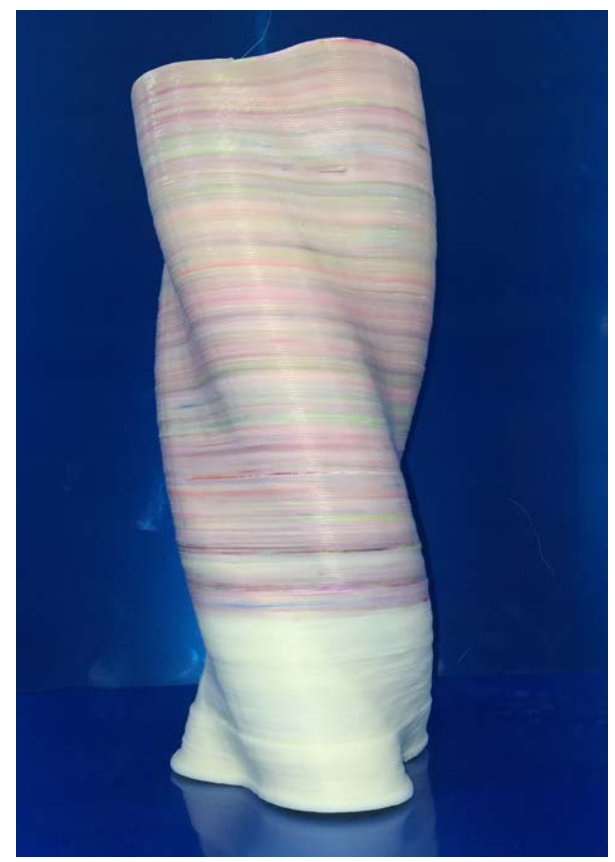

Figura 7: Las mezclas de materiales otorgan flexibilidad en colores, acabados y propiedades de los materiales. El formato de pellet es mucho más adecuado para lograr estas mezclas.

\section{ATASCOS EN EL SISTEMA}

Se logró identificar dos tipos de atasco en el sistema. El primer tipo fue un atasco mecánico entre alguna partícula y el orificio de entrada del tubo con el husillo. Este tipo de atasco se logró erradicar con una apertura de entrada al tubo mayor, así como un tamaño de partícula menor. Las partículas menores a $2 \mathrm{~mm}$ son las ideales para esto. Otro tipo de atasco es el fenómeno conocido en inglés como "jamming" (Whyman, Arif, \& Potgieter, 2018) que sucede naturalmente en agregados granulares sujetos a compresión. Este fenómeno ha sido definido como una transición de fase en materiales granulares ya que presenta propiedades similares a los semilíquidos al transmitir presiones en todas direcciones, opuestamente a los sólidos que transmiten fuerzas en la dirección de la fuerza aplicada. Este fenómeno, una vez más, se minimiza con un diámetro de partícula menor.

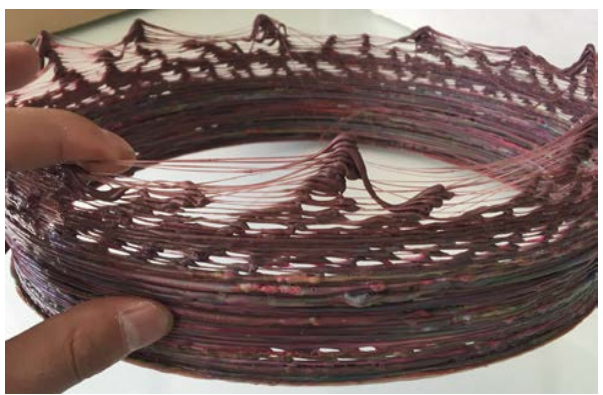

Figura 8: Atascos en el sistema se deben mayoritariamente al jamming, y la optimización de tamaño de partícula, temperatura de extrusión y material mitiga este efecto.

\section{RESISTENCIA DE LOS OBJETOS}

Los objetos impresos muestran buena resistencia a cargas axiales como se demuestra en pruebas de funcionalidad para objetos de mobiliario. Debido a la lógica de fabricación, los objetos impresos son más resistentes en el sentido perpendicular a la impresión de las capas.

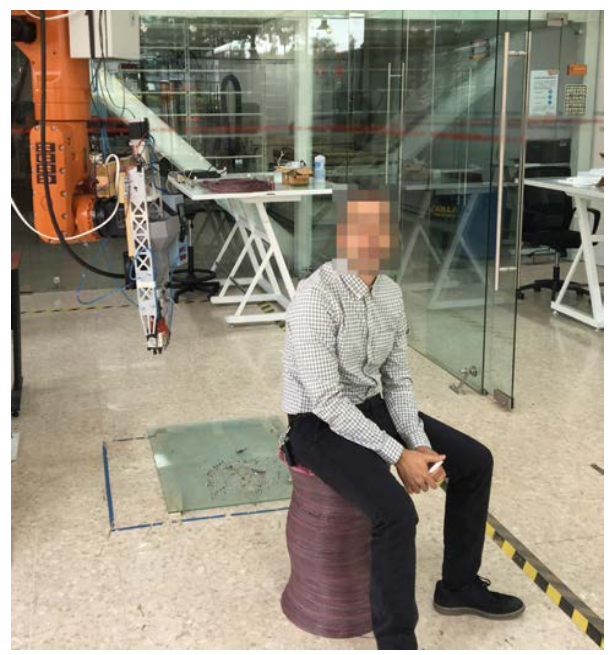

Figura 9: El objeto impreso es funcional como artefacto de mobiliario.

\section{ENCOGIMIENTO TÉRMICO Y CÓMO MITIGARLO}

Uno de los mayores problemas con los que se encuentra cualquier persona que desee hacer impresión FDM de gran formato es el encogimiento térmico. El plástico, al estar en su fase plástica, que es en temperaturas de arriba de los $170^{\circ} \mathrm{C}$ expande su volumen por dilatación térmica. Al ser extruido por el dispositivo en esta fase y enfriarse después de un tiempo, el plástico comienza a contraerse debido a que su temperatura baja. Este fenómeno es común en todas las impresoras FDM, pero se exacerba a la hora de imprimir con los diámetros de boquilla y alturas de capa que proponemos. Adicionalmente, existen materiales que son especialmente sensibles a este fenómeno. Nuestra experiencia con PLA fue mucho mejor que con $A B S$. El plástico ABS presenta encogimientos dramáticos al tener un coeficiente de contracción alto.

Para mitigar este efecto se utilizaron varias estrategias, algunas desde la operación y diseño del dispositivo y otras desde la creación de la geometría a imprimir. La primera estrategia fue la de mantener el área de impresión a una temperatura mayor a la ambiente, para evitar enfriamientos diferenciales. Se cubrió todo el sistema con una cubierta de bajo costo a base de plástico de polietileno de alta densidad, para poder mitigar los impactos de la temperatura ambiental. Adicionalmente se diseñó e implementó una cama caliente a base de resistencias eléctricas y cristal templado, lo que permite una mejor adhesión a la cama de impresión y un enfriamiento constante a los largo de toda la superficie. La temperatura de extrusión juega un papel importante ya que es mucho mejor imprimir a temperaturas bajas alrededor de los $190^{\circ} \mathrm{C}$ para $\mathrm{PLA}$ y $205^{\circ} \mathrm{C}$ para $\mathrm{ABS}$. 
Adicionalmente, la geometría de los objetos diseñados, pueden ser optimizados para mitigar los efectos del encogimiento. La simetría radial en el diseño de los objetos tiene efectos positivos, ya que si el encogimiento se presenta, este es repartido en partes iguales alrededor de los contornos de impresión, lo que evita desplazamientos que estropeen la impresión. La topología cilíndrica de los objetos diseñados contribuye a esta repartición del encogimiento térmico, sobretodo en capas a menor altura, más cercanas a la cama de extrusión.

Por último, y aunado a las estrategias previamente descritas, se optó por la inclusión de una pistola de aire caliente común que apuntada hacia la boquilla de extrusión ayuda a que el material tenga una temperatura uniforme a lo largo del objeto, evitando encogimientos diferenciales. Un efecto positivo de esta última estrategia, es que el aire caliente permite que la capa anterior a la trayectoria de extrusión sea calentada previo a recibir el plástico extruido, mejorando la adherencia entre capa y capa.

\section{CONCLUSIONES}

Hemos discutido el diseño y operación del dispositivo, los criterios y parámetros requeridos para el diseño de objetos con nuestro sistema, pero es necesario discutir sus implicaciones y posibles aplicaciones en la construcción y el diseño de espacios arquitectónicos.

Este trabajo pretende compartir nuestra experiencia con el desarrollo del dispositivo, el desarrollo de las trayectorias, y el diseño de los objetos de escala meso para diseñadores y arquitectos interesados en la impresión $3 \mathrm{~d}$ de objetos de escala arquitectónica, sin embargo, la escala meso, es un paso obligado para lograr que la tecnología FDM e implementada en la escala del edificio. Antes de que la tecnología FDM sea implementada a la escala arquitectónica, es necesario primero dominar la escala meso. Las aplicaciones de esta tecnología, en escala meso, pueden ser componentes agregados para cimbras de concreto, mobiliario, y sistemas de fachada, etc. El uso de plástico reciclado es un componente clave de nuestro sistema de trabajo ya que puede ser una manera de usar al diseño como fuerza transformadora de los modelos de consumo de las comunidades de creadores, universidades, y laboratorios. Al cerrar ciclos de consumo y buscar el uso de material de desperdicio en objetos funcionales, nuestra sociedad puede transformar modelos extractivos y aumentar el ciclo de vida de los materiales que usamos todos los días.

La tecnología FDM tiene aún muchos retos para lograr su implementación en la escala arquitectónica, pero proyectos como este pueden ir avanzando la tecnología para lograr una integración de esta tecnología en el sitio de la construcción. Hemos identificado dos retos principales para implementar esta tecnología en sitios de construcción: el encogimiento térmico y las velocidades de impresión. El encogimiento térmico es el reto más importante para la impresión 3d a gran escala de materiales termoplásticos; implementar sistemas de control para mitigar este efecto es esencial para lograr impresiones consistentes. Por ende, el uso de impresión $3 d$ de termoplásticos in-situ en ambientes de construcción puede no ser viable si no se considera ambientación del sitio. Las velocidades de impresión sólo son viables con alturas de capa mayores a $1 \mathrm{~mm}$, ya que menores alturas toman demasiado tiempo para poder monitorear el desarrollo de la impresión. El uso de filamento de extrusión no es viable para las velocidades de impresión y los tamaños de boquilla utilizados, por lo que los pellets de plástico deben de ser el futuro de la impresión FDM.

El uso de este sistema, también requiere de un control altamente específico de las trayectorias de impresión, razón por la cual herramientas comerciales no son suficientes para poder utilizar nuestro sistema. Dichas herramientas tienen controles automatizados para cálculo de trayectorias, pero con la automatización, se pierde el control de las trayectorias que en un ambiente de iteración y diseño de dispositivos simplemente no permite la optimización del sistema. Probablemente en un futuro el sistema permita generar herramientas de software con los parámetros idóneos internalizados, mismos que emanarán de amplias experimentaciones de las cuales nuestro trabajo es un solo ejemplo de muchos.

Por último, existe una estrecha relación entre el funcionamiento y limitantes del sistema, y el diseño de objetos y trayectorias. Por esta razón se potencia el papel del diseñador no sólo como usuario de un sistema sino como creador del sistema mismo. Cada vez más, la división entre creadores usuarios, e ingenieros y desarrolladores es más difusa, y la construcción de máquinas es sin duda una habilidad clave en el banco de herramientas del arquitecto y diseñador contemporáneo. Debemos convertirnos en generadores de tecnología y creadores de máquinas si deseamos llevar hacia adelante la profesión; pasar de usuarios de tecnología a creadores de tecnología. Esta habilidad no sólo permite la adherencia a principios físicos importantes en la cultura material, sino que permite la liberación del diseñador como creador y constructor, a través de la tecnología como medio más que como herramienta. Esta es una discusión más extensa, pero que a través de nuestro trabajo apuntamos a un modelo de re-pensamiento transformador del papel, las habilidades del diseñador, y su impacto en los ciclos de consumo material en su comunidad.

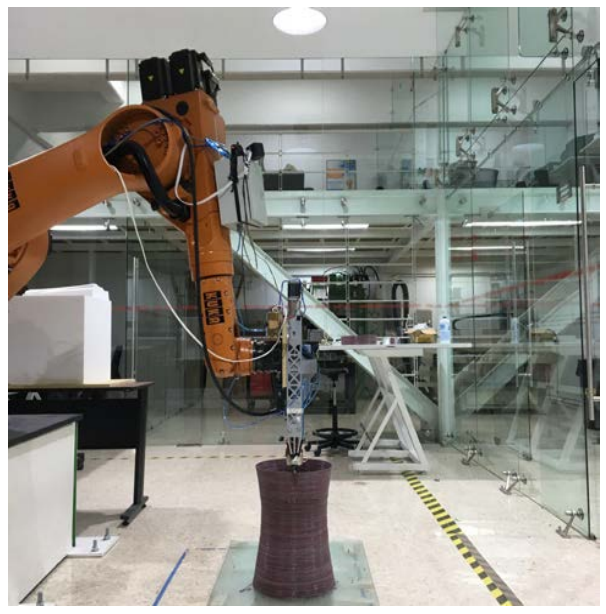

Figura 10: Dentro de los laboratorios de fabricación digital, es fundamental comenzar a generar nuevas implementaciones de las tecnologías a la mano. 


\section{AGRADECIMENTOS}

El mayor agradecimiento debe de ser a mi compañero durante todo este proceso y coautor de la tecnología presentada, al D.I. Rafael Ramos Méndez. Este trabajo se realizó con el apoyo de muchas personas. Agradezco especialmente al Dr. Bernardo Gómez-Pimienta, Director de la Facultad de Arquitectura de la Universidad Anáhuac México, y al Dr. Gonzalo Pérez Ramírez, Coordinador de Fablab México, sin su apoyo este trabajo no hubiera sido posible. Se agradece también a los Ingenieros Florencio Marín, e Iván Hernández quienes apoyaron durante largas horas en este proceso.

\section{REFERENCIAS}

Whyman, S., Arif, K., \& Potgieter, J. (2018). Design and development of an extrusion system for 3D printing biopolymer pellets. International Journal of Advanced Manufacturing Technology (96), 3417-3428.

Borden, G. P., \& Meredith, M. (2012). Matter: Material Processes in Architectural Production. Londres: Routledge.

Rael, R., \& San Fratello, V. (2018). Printing Architecture: Innovative Recipes for $3 D$ Printing. Nueva York: Princeton Architectural Press.

Asociation for Robots in Architecture. (2020, 04 05). Kuka Parametric Robot Control. Retrieved from https://www.robotsinarchitecture.org/kuka-prc

Ingrassia, D. (2020, 07 01). Big FDM Retrieved from https://github.com/fab-machines/BigFDM 